\title{
Antenatal Corticosteroid Exposure As a Risk Factor For Neonatal Hyperbilirubinemia
}

\author{
El-Hawary MA ${ }^{1}, \mathrm{Al}$ Gameel KA ${ }^{2}$, El- Garhy SAAS ${ }^{3}$, \\ 1. Professor of pediatric, Faculty of Medicine, Fayoum University. \\ 2. Professor of pediatric, Faculty of medicine, Fayoum University. \\ 3. M.B.B.CH, Faculty of medicine, Fayoum university, Egypt.
}

\section{Abstract:}

Introduction: Jaundice is observed during first week of life in approximately $60 \%$ of term neonates and $80 \%$ of preterm neonates. Untreated severe unconjugated hyperbilirubinemia is potentially neurotoxic. In the last few years, randomised controlled trials (RCT) have demonstrated significant improvement in respiratory morbidities even in late preterm infants when antenatal corticosteroids (ACS) was administered. However, administration of ACS is not without complications.

Patients and Methods: This study included 200 neonates with indirect hyperbilirubinemia in phototherapy range. Neonates were divided into 2 groups according to exposure to ACS. All patients were subjected to a full medical history, full clinical examination, blood sample for daily assessment of serum total and direct bilirubin level, complete blood count (CBC) examination, reticulocyte count, maternal, infant blood groups $\mathrm{ABO}$ and $\mathrm{Rh}$.

Jaundice is observed during first week of life in approximately $60 \%$ of term neonates and $80 \%$ of preterm neonates. Untreated severe unconjugated
Results: This study showed that, (68.5\%) of cases with neonatal hyperbilirubinemia, received ACS and (31.5\%) did not receive it. More than half of the studied cases were males (57\%), females constituted (43\%). The onset of jaundice in the majority of cases $(36.5 \%)$ were at $3 \mathrm{rd}$ day of age, the rest of cases were between 4th to 8th day of age. There was no statistically significant difference in mean birth weight between both groups.

Conclusion: The administration of antenatal corticosteroid has no significant effect on increasing incidence of neonatal hyperbilirubinemia. Also we found that there was no clear indications for ACS administration among the majority of exposed cases.

Key words: Randomised controlled trials, Antenatal corticosteroids, Complete blood count.

\section{Introduction:}

hyperbilirubinemia is potentially neurotoxic [1].

In the last few years, randomised controlled trials (RCT) have 
demonstrated significant improvement in respiratory morbidities even in late preterm infants when antenatal corticosteroids (ACS) was administered [2].

However, administration of ACS is not without complications. Systematic reviews of literature involving animal and human studies have reported that impaired glucose tolerance, hypertension. and a reduction in brain growth after exposure to ACS administration. This has generated recent discussion about the risk and benefit of ACS administration, especially in view of long-term adverse outcomes [3]. In addition, ACS may also contribute to another important metabolic complication for preterm infants as hyperbilirubinemia. An observational study of 34 preterm infants showed that those exposed to antenatal dexamethasone had higher levels of serum unconjugated bilirubin for the first week after birth compared to nonexposed controls as well as a higher rate of hyperbilirubinemia requiring treatment [4].

\section{Aim of the work:}

The aim of the work is to evaluate the effect of antenatal corticosteroid administration as a risk factor in the development of neonatal hyperbilirubinemia..

\section{Patient and methods:}

This cross-sectional study was conducted in neonatal intensive care unit of Fayoum University Hospital and neonatal intensive care unit of Fayoum Health Insurance Hospital. It included 200 neonates with indirect hyperbilirubinemia in phototherapy range. Neonates were divided into 2 groups according to exposure to antenatal corticosteroid:

Group (A): 137 neonates exposed to antenatal corticosteroid.

Group (B): 63 neonates not exposed to antenatal corticosteroid.

All patients were subjected to a full medical history, full clinical examination, blood sample for daily assessment of serum total and direct bilirubin level, complete blood count (CBC) examination, reticulocyte count, maternal, infant blood groups $\mathrm{ABO}$ and $\mathrm{Rh}$.

\section{Statistical analysis of data:}

The collected data were organized, tabulated and statistically analyzed using Statistical Package for the Social Science (SPSS) software statistical computer package version 18 (SPSS Inc, USA). Quantitative data was presented as mean, standard deviation (SD) and range. Independent t test was used as a test of significance. Qualitative data were presented as frequencies and percentages, chi square $(\chi 2)$ was used as a test of significance. For interpretation of results of tests of significance, significance was adopted at $\mathrm{P} \leq 0.05$. 


\section{Results:}

This study was conducted on 200 neonates with indirect hyperbilirubinemia in phototherapy range. The mean age of neonates in our study was $(5 \pm 1.8)$ days ranged between 3-10 days. More than half of them were males $(57 \%)$, females constituted (43\%).

Regarding the history of antenatal corticosteroid (ACS) administration (68.5\%) of studied cases received ACS and $(31.5 \%)$ did not receive it. The mean gestational age was $(37.2 \pm 0.9)$ weeks, ranged between 35-39weeks. The majority of studied cases were full term (77.5\%), preterm deliveries constituted (22.5\%) as shown in table 1.

Regarding indication for ACS administration, almost half of our cases who received ACS (49\%) had no clear indication. The indication for ACS administration in the other half of our cases were elective CS in (17.5\%), premature contraction in $(11.7 \%)$ of cases, previous spontaneous preterm birth in $(6.5 \%)$ and oligohydraminos in $(15.3 \%)$ of cases.

Regarding the onset of jaundice, the majority of cases (36.5\%) were at 3rd day of age, the rest of cases were between 4th to 8th day of age.
Although the number of cases who developed jaundice on the 3rd day of life was larger among those who received ACS than those who didn't ( 51 vs 22) the difference did not reach a statistical significance $P$ value 0.796 as shown in table 2.

There was no statistically significant difference in serum total and direct bilirubin level values on admission and on discharge between neonates exposed to ACS and those without exposure as shown in table 3 .

Regarding mode of delivery, The majority of neonates exposed to Corticosteroids antenatally $96.4 \%$ were delivered by CS compared to those $74.6 \%$ not exposed with a statistically significant difference with P-value $<0.0001$ as shown in figure 1 .

However, regarding birth weight and maternal age there was no statistically significant difference between neonates exposed to ACS or not.

\section{Discussion:}

This study included 200 neonates with indirect hyperbilirubinemia in phototherapy range aiming to clarify the relation between antenatal corticosteroid administration and neonatal hyperbilirubnemia..

Our results showed that, out of all studied cases 200 neonates with indirect 
hyperbilirubinemia in phototherapy range, 137 neonates (68.5\%), received antenatal corticosteroid. While only 63 neonates $(31.5 \%)$, did not receive it. Among all the studied cases the onset of jaundice was between 3rd and 8th day of life. Although the number of cases who developed jaundice was larger among those who received ACS than those who did not, especially among those who developed jaundice on the 5th day, the difference did not reach a statistical significant difference. Also in our study, we found no statistical significant difference in serum bilirubin level values on admission and on discharge between cases exposed to ACS and those without exposure.

These results disagree with $(\mathrm{Ne}$ 'meth , et al 1981), observational study of 34 preterm infants showed that those exposed to antenatal dexamethasone had higher levels of serum unconjugated bilirubin for the first week after birth compared to unexposed controls as well as a higher rate of hyperbilirubinemia requiring treatment ${ }^{[5]}$.

Also this result disagree with (Kate et al., 2014), which showed that newborns exposed to antenatal corticosteroid had higher rate of incidence of hyperbilirubinemia and found an association between antenatal betamethasone exposure and neonatal hyperbilirubinemia ${ }^{[4]}$.
In contrast a study by Liggins and Howie in 1972 (Liggins, et al 1972), Administration of antenatal corticosteroids to mothers at high risk for preterm birth has been shown to markedly improve neonatal outcomes and showed no increase in rates of hyperbilirubinemia in the betamethasone exposed infants ${ }^{[6]}$.

In another study, the neonatal hyperbilirubinemia was significantly decreased in term-born babies exposed to ACS before 34 gestational weeks. The neonatal hyperbilirubinemia rate was 20/354 (5.6\%) in the ACS treatment group and 564/5900 (9.6\%) in the control group (Madendag et al ., 2019) ${ }^{[7]}$.

In our study, more than half of the studied cases were males (57\%), females constituted (43\%). This agree with the study conducted in AL -Azhar University, Assuit, Egypt. males represented $80 \%$ of cases with nonhemolytic jaundice (Ahmed et al., 2019) [8].

Similarly, carried out in NICU at AL Ramadi teaching hospital for maternity and childhood, Iraq, where $(60.4 \%)$ of jaundiced neonates were males and (39.6\%) were females (Shitran et al., 2020) ${ }^{[9]}$

In contrast, Venkatamurthy et al., 2014 study of 98 male and 76 female babies and showed that, the neonatal hyperbilirubinemia $\quad(>17 \mathrm{mg} / \mathrm{dl}) \quad$ is independent of the sex of the neonate ${ }^{[10]}$. 
In our study, regarding the mean maternal age there was no statistically significant difference between neonates exposed to ACS or not. This came in agree with Ramadan et al., 2016 who found that there is no significant difference in the mean maternal age between exposed and non-exposed groups ${ }^{[11]}$.

Similarly, in a tertiary academic centre hospital, Canada, there was no significant differences in the mean maternal age between exposed (32.3 \pm $6.3)$ and un-exposed (33.4 \pm 4.7$)$ groups (Jones et al., 2020) ${ }^{[12]}$.

Our results disagree with (Kate et al 2014), which showed increased rates of neonatal hyperbilirubinemia with betamethasone administration three times more with maternal age $>35$ years ${ }^{[4]}$.

\section{Limitations:}

better include a larger scale of patients.

\section{Conclusion:}

From this work we concluded that administration of antenatal corticosteroid has no significant effect on increasing incidence of neonatal hyperbilirubinemia. Also we found that there was no clear indications for ACS administration among the majority of exposed cases.

\section{References:}

[1]: Kliegman RM.(2015): Jaundice and Hyperbilirubinemia in the Newborn. In Behrman R, Nelson Text book of Pediatrics. 20th ed. Philadephia:Elsevier,871-875.

[2]: Gyamfi-Bannerman, C., Thom, E. A., Blackwell, S. C., Tita, A. T., Reddy, U. M., Saade, G. R., ... \& Chien, E. K. (2016): Antenatal betamethasone for women at risk for late preterm delivery. New England Journal of Medicine, 374(14), 1311-1320.

[3]: Srinivasjois, R., \& Silva, D. (2017): Antenatal steroid administration in medically uncomplicated pregnancy beyond 37 weeks of gestation for the prevention of neonatal morbidities prior to elective caesarean section: a systematic review and meta-analysis of randomised controlled trials. The Journal of Maternal-Fetal \& Neonatal Medicine, 30(10),1151-1157.

[4]:Kate E. Pettit , Susan H. Tran , Erin Lee, et al ( 2014): The association of antenatal corticosteroids with neonatal hypoglycemia and hyperbilirubinemia $\mathbf{J}$ Matern Fetal Neonatal Med 10.3109/14767058.2013.832750.

[5]: Ne 'meth, I., Szeleczki, T., \& Boda, D.( 1981): Hyperbilirubinemia and urinary D-glucaric acid excretion in premature infants following antepartum 
dexamethasone treatment. $\mathrm{J}$ Perinat Med,9(5-9),3.

[6]:.Liggins GC, Howie RN.,(1972) :A controlled trial of antepartum glucocorticoid treatment for prevention of the respiratory distress syndrome in premature infants. Pediatrics 1972;50:515-25.

[7]: Madendag, I. C., \& Sahin, M. E. (2019): The effects of antenatal corticosteroid exposure on the rate of hyperbilirubinemia in term newborns. Pakistan Journal of Medical Sciences, 35(6), 1582.

[8]: Ahmed, Y. A., Kassem, Y. T., \& Ismail, S. A. N. (2019): Effect of Intensive Phototherapy on Bilirubin Induced Neurological Defect (BIND) Score in Neonates with Severe Hyperbilirubinemia. The Egyptian Journal of Hospital Medicine, 74(7), 1643-1648.

[9]: Shitran RF, Abed MY (2020): Risk factors and outcome of neonatal jaundice Table (1): Prenatal and natal history of the studied cases $(\mathrm{N}=200)$

\begin{tabular}{|c|c|c|}
\hline Variable & Mean \pm SD & Range \\
\hline Gestational Age (Weeks) & $37.2 \pm 0.9$ & $35-39$ \\
\hline Variable & Number & $(\%)$ \\
\hline \multicolumn{3}{|l|}{ History of ACS } \\
\hline No & 63 & $31.5 \%$ \\
\hline Yes & 137 & $68.5 \%$ \\
\hline
\end{tabular}




\begin{tabular}{|c|c|c|}
\hline Full term & 155 & $77.5 \%$ \\
\hline Preterm & 45 & $22.5 \%$ \\
\hline \multicolumn{3}{|c|}{ Mode of delivery } \\
\hline $\mathrm{CS}$ & 179 & $89.5 \%$ \\
\hline NVD & 21 & $10.5 \%$ \\
\hline
\end{tabular}

ACS: Antenatal corticosteroid.

CS: caesarean section.

NVD: Normal vaginal delivery.

Table (2): Comparison of onset of jaundice between neonates with ACS and those without

\begin{tabular}{|c|c|c|c|c|c|}
\hline & \multicolumn{4}{|c|}{ ACS } & \multirow{3}{*}{ P-value } \\
\hline & \multicolumn{2}{|c|}{$\begin{array}{c}\text { No } \\
(\mathbf{N}=63)\end{array}$} & \multicolumn{2}{|c|}{$\begin{array}{c}\text { Yes } \\
(\mathbf{N}=137)\end{array}$} & \\
\hline & $\mathbf{N}$ & $\%$ & $\mathbf{N}$ & $\%$ & \\
\hline \multicolumn{6}{|c|}{$\begin{array}{l}\text { Onset of jaundice } \\
\text { (days) }\end{array}$} \\
\hline $3^{\text {rd }}$ day & 22 & $34.9 \%$ & 51 & $37.2 \%$ & \multirow{6}{*}{0.796} \\
\hline $4^{\text {th }}$ day & 21 & $33.3 \%$ & 38 & $27.7 \%$ & \\
\hline $5^{\text {th }}$ day & 8 & $12.7 \%$ & 28 & $20.4 \%$ & \\
\hline $6^{\text {th }}$ day & 7 & $11.1 \%$ & 12 & $8.8 \%$ & \\
\hline $7^{\text {th }}$ day & 4 & $6.3 \%$ & 6 & $4.4 \%$ & \\
\hline $8^{\text {th }}$ day & 1 & $1.6 \%$ & 2 & $1.5 \%$ & \\
\hline
\end{tabular}

Table (3): Comparison of bilirubin levels in neonates both exposed and non-exposed to $\operatorname{ACS}(\mathbf{N}=200)$

\begin{tabular}{|c|c|c|c|c|c|}
\hline \multirow{3}{*}{ Variable } & \multicolumn{4}{|c|}{ ACS } & \multirow{3}{*}{ P-value } \\
\hline & \multicolumn{2}{|c|}{$\begin{array}{c}\text { No } \\
(\mathbf{N}=63)\end{array}$} & \multicolumn{2}{|c|}{$\begin{array}{c}\text { Yes } \\
(\mathbf{N}=137)\end{array}$} & \\
\hline & Mean & $\pm \mathrm{SD}$ & Mean & \pm SD & \\
\hline \multicolumn{6}{|l|}{$\begin{array}{l}\text { Total bilirubin } \\
(\mathrm{mg} / \mathrm{dl})\end{array}$} \\
\hline On admission & 17.63 & 1.34 & 17.47 & 1.36 & 0.437 \\
\hline On discharge & 7.61 & 0.97 & 7.63 & 0.94 & 0.845 \\
\hline
\end{tabular}


ISSN: 2536-9482 (Online) Fayoum University Medical Journal El-Hawary et al., 2020,8(4), 12-

\begin{tabular}{|c|c|c|c|c|c|}
\hline \multicolumn{6}{|l|}{$\begin{array}{l}\text { Direct bilirubin } \\
\text { (mg/dl) }\end{array}$} \\
\hline On admission & 0.97 & 0.37 & 0.92 & 0.33 & 0.297 \\
\hline On discharge & 0.58 & 0.18 & 0.56 & 0.17 & 0.391 \\
\hline
\end{tabular}

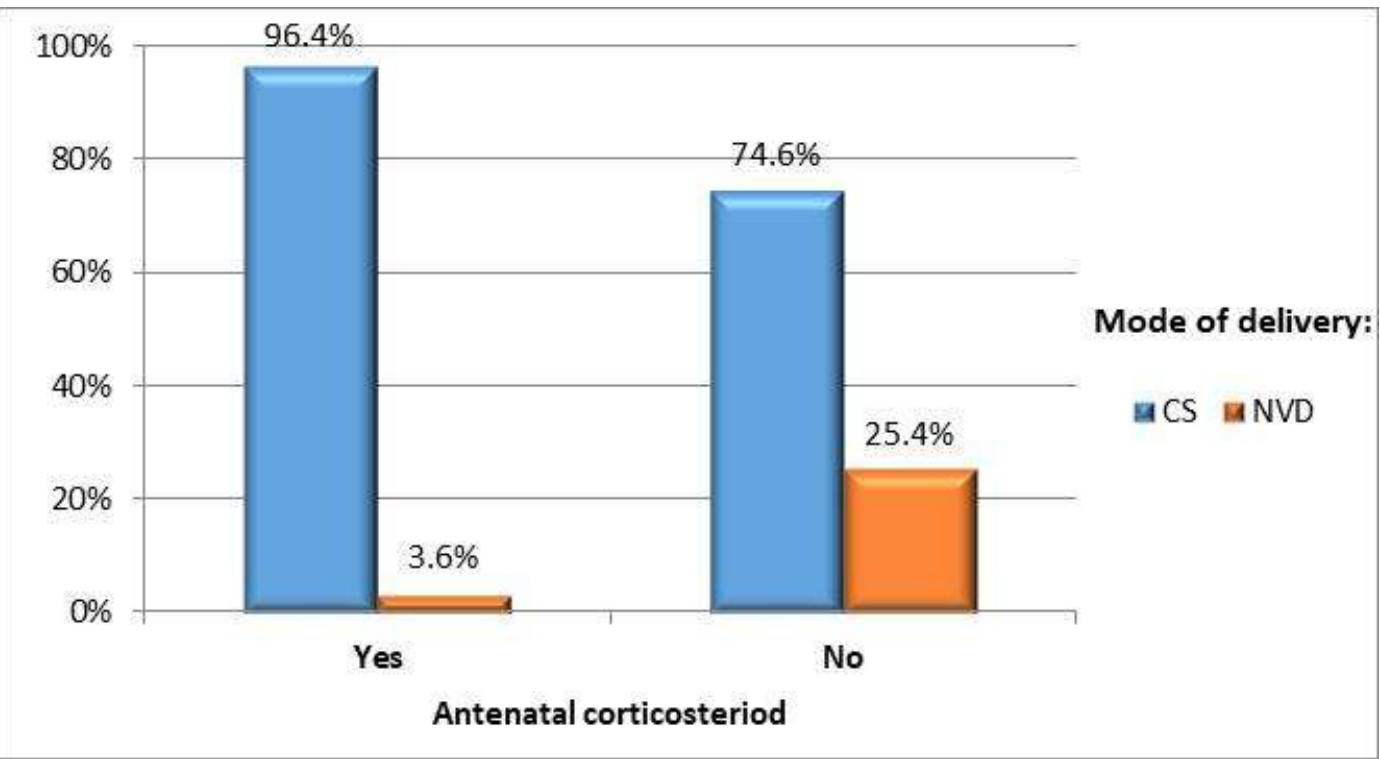

Figure (1): comparison between neonates exposed to ACS and not, regarding mode of delivery. 